UVX 2012, 01015 (2013)

DOI: $10.1051 / \mathrm{uvx} / 201301015$

(C) Owned by the authors, published by EDP Sciences, 2013

\title{
L'effet phare attoseconde
}

\author{
H. Vincentia et F. Quéréb
}

CEA Saclay, Service des Photons Atomes et Molécules, 91190 Gif-sur-Yvette, France

\begin{abstract}
Résumé. Lorsqu'on focalise un laser intense dans un gaz ou un plasma, un train d'impulsions attosecondes associé à un spectre d'harmoniques du champ incident est généralement émis. Dans cet article, on présente une toute nouvelle approche à la génération d'impulsions attosecondes isolées, qui consiste à envoyer les impulsions attosecondes du train dans des directions différentes, à la manière d'un "phare », puis d'en filtrer spatialement une seule à l'aide d'une fente placée en champ lointain [1].
\end{abstract}

\section{Principe}

Depuis l'avènement de la Science Attoseconde dans les années 1970, de nombreux efforts ont été fournis pour générér des impulsions attosecondes isolées, qui sont en général plus appropriées au cas d'expériences résolues en temps. Les techniques qui ont été développées jusqu'alors pour résoudre ce problème (fenêtrage temporel par intensité ou par polarisation), ont pour principe d'éliminer toutes les impulsions du train sauf une. Comme l'illustre la Fig.1, on adopte ici une approche totalement nouvelle qui consiste plutôt à disperser les impulsions du train en angle puis à en sélectionner une seule à l'aide d'un masque spatial. Une manière simple d'obtenir cette dispersion angulaire des impulsions attosecondes est de disposer d'un champ dont les fronts de phase tournent dans le temps au foyer, là où a lieu la génération d'harmoniques. En effet, dans le cas usuel où les fronts de phase du champ électrique sont parallèles, les impulsions attosecondes sont émises dans la même direction, donnée par la normale au front d'onde instantané du laser (cf. Fig.1). En présence d'une rotation du front d'onde (abrégé en WFR pour wavefront rotation) et en raison de la cohérence du processus de génération d'harmoniques, les impulsions attosecondes serons émises dans des directions légèrement différentes au fil du temps (cf. Fig.1 (b)). Nous avons baptisé cet effet, «l'effet phare attoseconde ». Si la vitesse de rotation est suffisante, il devient alors possible de séparer correctement les impulsions en angle puis d'en isoler une seule à l'aide d'une fente placée en champ lointain. Dans la suite, on détaille comment il est possible d'obtenir du WFR au foyer d'un laser.

\section{Du tilt du front d'intensité à la rotation de front d'onde}

Avant focalisation, la rotation de front d'onde prend une forme totalement différente appelée tilt du front d'intensité (abrégé en PFT pour Pulse Front Tilt en anglais) [2], qui correspond à un champ électrique de la forme :

$$
E\left(x_{i}, t\right)=E_{0} \exp \left(-2\left[\frac{t-\xi x_{i}}{\tau_{i}}\right]^{2}-2 \frac{x_{i}^{2}}{w_{i}^{2}}\right) \times \exp \left(i \omega_{L} t\right)
$$

\footnotetext{
a e-mail : henri.vincenti@cea.fr

be-mail : fabien.quere@cea.fr

This is an Open Access article distributed under the terms of the Creative Commons Attribution License 2.0, which permits unrestricted use, distribution, and reproduction in any medium, provided the original work is properly cited.
} 
(a)

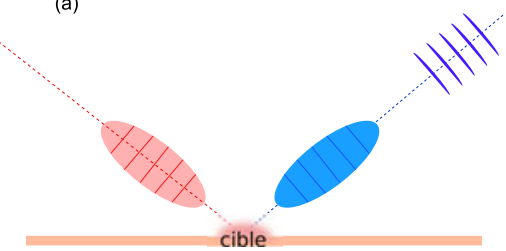

(b)

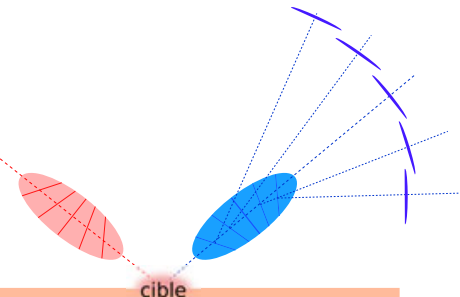

Figure 1. Principe de l'effet phare attoseconde. (a) Génération d'impulsions attosecondes par une impulsion laser classique dont les fronts d'onde sont parallèles. (b) Génération d'impulsions attosecondes à l'aide d'une impulsion possédant des fronts de phase qui tournent dans le temps.

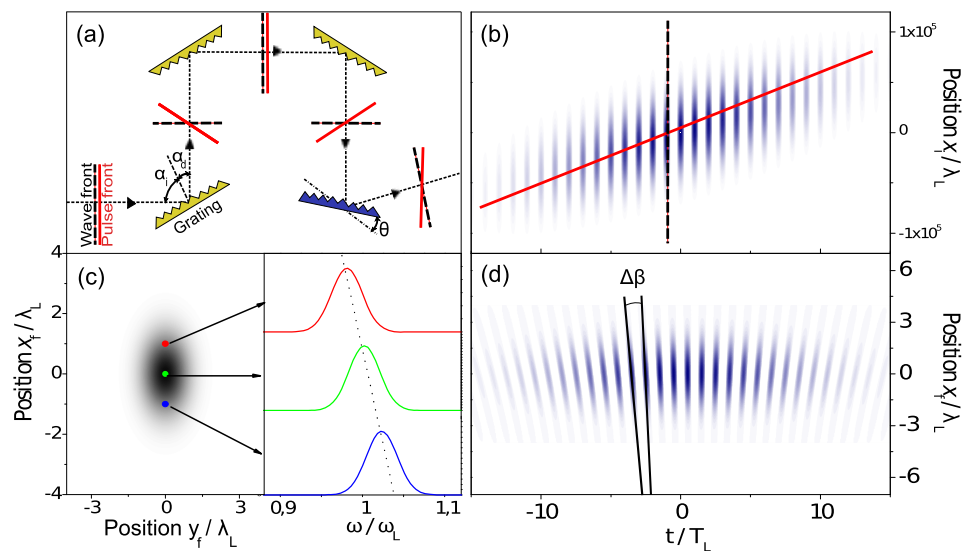

Figure 2. Tilt du front d'intensité et rotation de front d'onde d'un faisceau laser femtoseconde (a) illustre comment un compresseur à 4 réseaux légèrement désaligné peut conduire à du PFT résiduel à sa sortie. (b) Champ électrique $E$ [partie réelle e l'Eq. (1)] possédant un tilt de son front d'intensité. La couleur bleue correspond à $E>0$ et la couleur blanche à $E<0$. (c) Distribution d'intensité $2 \mathrm{D}$ et spectre au foyer. La tâche focale est allongée et le spectre du champ varie spatialement le long du grand axe de l'ellipse. (d) Champ $E$ associé au foyer [partie réelle de l'Eq. (2)], possédant des fronts de phase qui tournent dans le temps. Les paramètres laser sont $\tau_{i}=25 \mathrm{fs}, w_{i}=70 \mathrm{~mm}$ et $\xi=\tau_{i} / w_{i}=0,36 \mathrm{fs} / \mathrm{mm}$. Avec $f=200 \mathrm{~mm}$, cela conduit à $w_{f}=2,6 \lambda_{L}, \tau_{f}=35 \mathrm{fs}$ et $v_{r}=7 \mathrm{mrad} / \mathrm{fs}$. Ces paramètres sont typiques de ceux d'un laser laser femtoseconde haute puissance.

où, $x_{i}$ est une des coordonnées transverses à la propagation du champ, $\tau_{i}$ la durée TF de l'impulsion à une position donnée dans le faisceau, $w_{i}$ le diamètre du faisceau avant focalisation, $\xi$ le paramètre de PFT et $\omega_{L}$ la fréquence centrale du laser. Toutes les largeurs sont définies à $1 / e$ en intensité.

Le tilt du front d'intensité d'une impulsion peut-être induit après diffraction de l'impulsion sur un réseau: comme l'angle d'incidence $\alpha_{i}$ et l'angle de diffraction $\alpha_{d}$ sont différents [cf. Fig.2 (a)], le chemin optique accumulé va varier avec $x_{i}$ et être à l'origine d'un délai variable entre le front d'onde et le front d'intensité. Dans le cas d'une chaîne laser CPA, un tilt du front d'intensité résiduel survient par exemple lorsque une des paires de réseaux utilisées dans le compresseur optique n'est pas strictement parallèle [cf. Fig.2 (a)]. Cet exemple permet de comprendre facilement la configuration du champ électrique $E$ une fois qu'un faisceau avec PFT est focalisé. En effet, un réseau suivi par un élément optique focalisant agit comme un spectromètre. Au foyer, un tel faisceau présente un spectre qui dépend de l'espace, comme illustré sur la Fig.2 (c). Cet effet est plus connu sous le nom de chirp spatial dans l'espace $\left(x_{f}, \omega\right)$, où $x_{f}$ est la coordonnée transverse au foyer et $\omega$ la fréquence de la lumière. Lorsque $\xi$ augmente, la tâche focale devient de plus en plus elliptique avec un grand axe le long de la direction du chirp spatial. [cf. Fig.2 (c)]. 


\section{UVX 2012}

Dans le domaine $\left(x_{f}, t\right)$, ce chirp spatial implique que l'espacement temporel entre deux fronts d'onde successifs varie avec la coordonnée transverse $x_{f}$ : par conséquent, les fronts d'onde tournent dans le temps [cf. Fig. 2(d)]. En effet, en calculant $\tilde{E}(k, t)$ la TF de $E\left(x_{i}, t\right)$ et en utilisant $k=k_{L} x_{f} / f$, on obtient le champ $\tilde{E}\left(x_{f}, t\right)$ au foyer d'une optique de focale $\mathrm{f}$ :

$$
\begin{aligned}
& \tilde{E}\left(x_{f}, t\right) \propto \exp \left(-2 \frac{t^{2}}{\tau_{f}^{2}}-2 \frac{x_{f}^{2}}{w_{f}^{2}}\right) \times \exp \left(i \varphi\left(x_{f}, t\right)\right) \\
& \varphi\left(x_{f}, t\right)=4 \frac{w_{i} \xi}{w_{f} \tau_{f} \tau_{i}} x_{f} t+i \omega_{L} t
\end{aligned}
$$

où la durée d'impulsion au foyer $\tau_{f}$ et la taille effective du waist $w_{f}$ le long de l'axe $x_{f}$, sont données par:

$$
\begin{gathered}
\tau_{f}=\tau_{i} \sqrt{1+\left(w_{i} \xi / \tau_{i}\right)^{2}} \\
w_{f}=w_{0} \sqrt{1+\left(w_{i} \xi / \tau_{i}\right)^{2}}
\end{gathered}
$$

avec $w_{0}=2\left(\lambda_{L} f / \pi w_{i}\right)$ le waist du faisceau au foyer lorsque $\xi=0$. La direction instantanée de propagation de la lumière $\beta(t)$ est donnée par $\beta \approx k_{\perp} / k_{L}$, où $k_{L}=\omega_{L} / c$ est le vecteur d'onde laser et $k_{\perp}=\partial \varphi / \partial x_{f}$ sa composante transverse. Le terme $x_{f} t$ dans $\varphi$ implique que $\beta$ et donc, la direction du front d'onde instantané, varient dans le temps avec une vitesse de rotation $v_{r}=d \beta / d t$ déduite de l'Eq. (2) :

$$
v_{r}=\frac{w_{i}^{2}}{f \tau_{i}^{2}} \frac{\xi}{1+\left(w_{i} \xi / \tau_{i}\right)^{2}} .
$$

Pour une durée $\tau_{i}$ donnée et une divergence $\theta_{L}=w_{i} / f$ du faisceau-impulsion, $v_{r}(\xi)$ atteint un maximum $v_{r}^{\max }=w_{i} / 2 f \tau_{i}$ pour une valeur $\xi_{0}=\tau_{i} / w_{i}$. Intuitivement, cela peut se comprendre en écrivant $v_{r}=\Delta \theta / \Delta T$ comme le rapport de l'angle $\Delta \theta$ que balaye le vecteur d'onde du champ sur la durée $\Delta T$ requis pour ce balayage. Le maximum de vitesse $v_{r}^{\max }$ est donc atteint lorsque la lumière balaye l'angle le plus grand possible à savoir la divergence laser $\Delta \theta=\theta_{L}$ durant la durée minimale que peut avoir l'impulsion, à savoir sa durée TF $\tau_{i}$. Avant focalisation, ce cas optimal correspond à un délai $\tau_{i}$ entre les deux extrémités du faisceau de taille $w_{i}$ alors qu'au foyer, on obtient $w_{f}=\sqrt{2} w_{0}$ et $\tau_{f}=\sqrt{3} \tau_{i}$ soit une réduction d'un facteur 2 seulement de l'intensité au foyer comparé au cas $\xi=0$.

\section{Critère de séparation des impulsions attosecondes}

Afin d'obtenir $N$ faisceaux harmoniques séparés en angle, chacun contenant une impulsion attoseconde unique, la rotation $\Delta \theta=v_{r} \Delta t$ du front d'onde pendant l'intervalle de temps $\Delta t$ entre l'émission de deux impulsions attosecondes successives doit être plus grand que la divergence $\theta_{n}$ d'un de ces faisceaux centrés autour de la fréquence $n \omega_{L}$ [cf. Fig.3]. Puisque pour des paramètres lasers donnés, $\Delta \theta$ ne peut excèder $v_{r}^{\max } \Delta t=\theta_{L} \Delta t / 2 \tau_{i}$, ceci est possible si la divergence du faisceau harmonique est suffisamment petite i.e. :

$$
\frac{\theta_{n}}{\theta_{L}} \leq \frac{1}{\alpha p N_{c}}
$$

où $N_{c} \geq N$ est le nombre de cycles optiques du laser incident, $p$ le nombre d'impulsions attosecondes générées à chaque cycle optique et $\alpha=O(1)$ un préfacteur qui dépend du contraste en intensité requis 


\section{Web of Conferences}

(L)

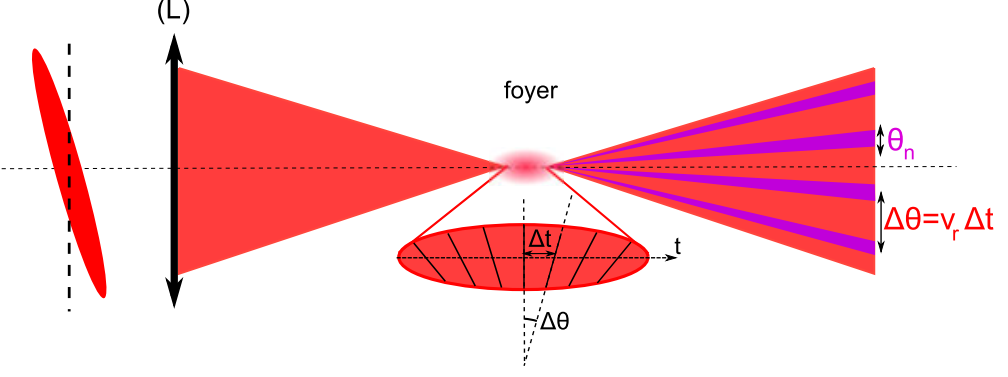

Figure 3. Critère de séparation des impulsions attosecondes.
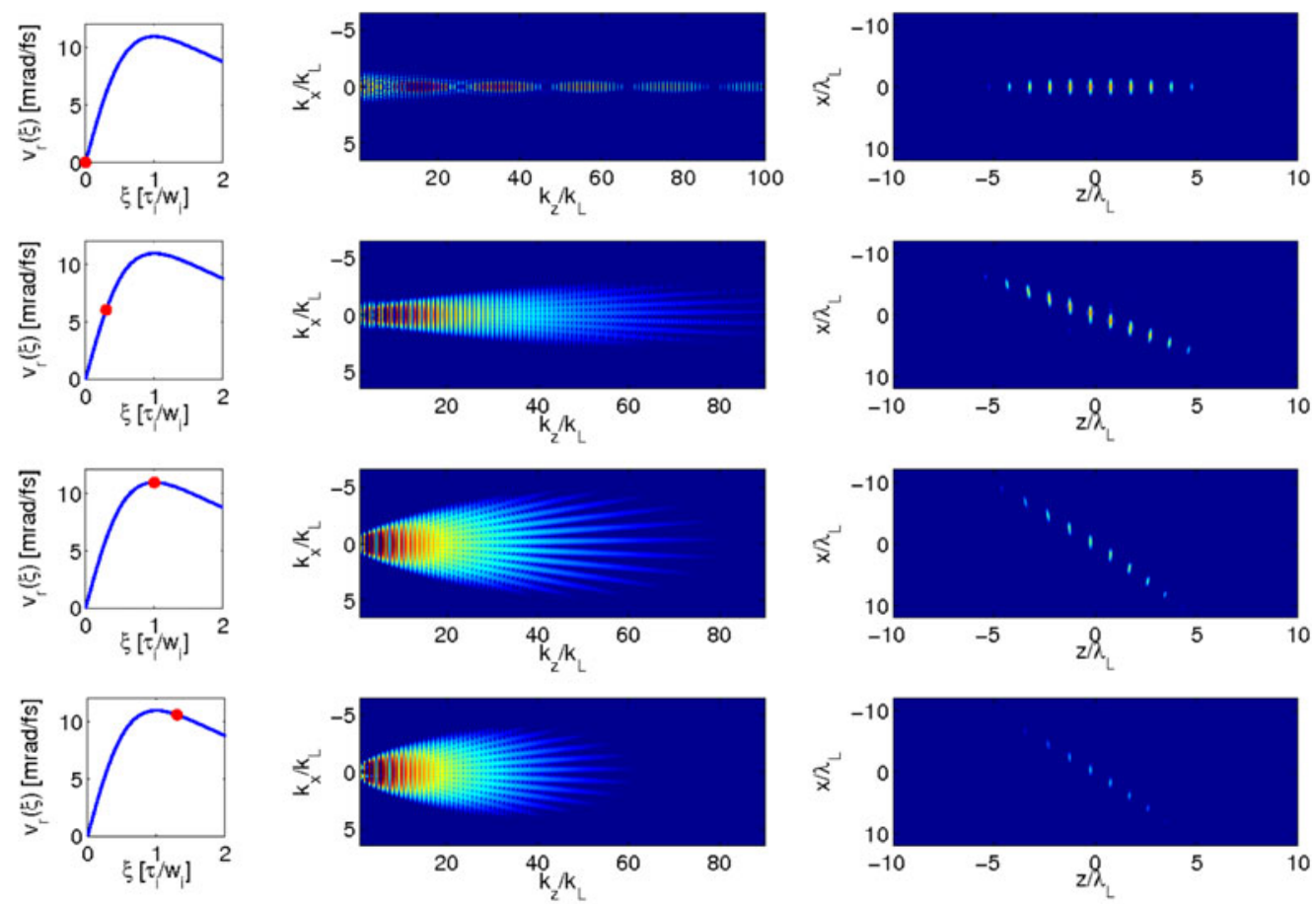

Figure 4. Résultats du modèle ROM appliqué à l'effet phare attoseconde. Les paramètres du faisceau laser sont les mêmes que ceux de la figure 2 pour un vecteur potentiel normalisé $a_{L}=6$. La première ligne correspond au cas $\xi=0$, la deuxième à $\xi=\tau_{i} / 2 w_{i}$ la troisième à $\xi=\tau_{i} / w_{i}$ (pour lequel on a une vitesse de rotation $v_{r}(\xi)$ maximale) et la dernière à $\xi=1.2 \tau_{i} / w_{i}$. Les panneaux de gauche montrent la vitesse de rotation des fronts d'onde du champ sur la cible. La deuxième colonne, elle, montre la transformée de Fourier à deux dimensions du champ $\widetilde{E}\left(x_{f}, t\right)$, après son interaction avec la cible. Dans ces graphes, la distance à l'origine correspond à la fréquence $\omega$ et l'angle polaire à la direction de propagation du champ. Enfin, les panneaux de droite montrent des instantanés du profil spatial d'intensité des impulsions attosecondes, obtenues après un filtrage du champ réfléchi entre les ordres harmoniques 70 et 80 , en fonction des coordonnées transverse $x$ et longitudinale $z$, à une distance de plusieurs longueurs de Rayleigh des harmoniques considérées.

entre une impulsion attoseconde donnée et ses satelliltes. L'Equation (5) nous donne la plus longue durée d'impulsion que l'on pourra utiliser pour générer des impulsions attosecondes correctement isolées en angle, pour un ratio donné entre la divergence harmonique et la divergence laser (qui est en général imposé par la physique du processus de génération et la configuration expérimentale). 

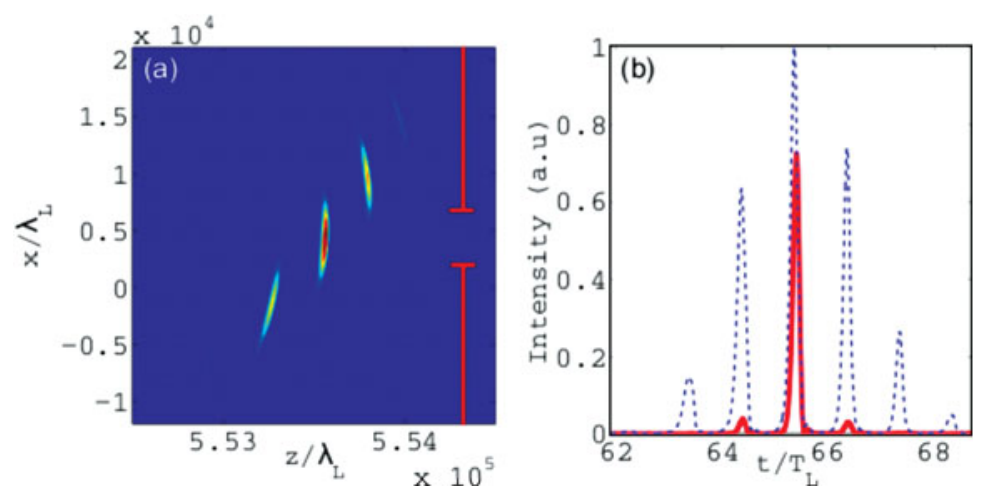

Figure 5. Simulation particulaire 2D de l'effet phare attoseconde. (a) Le panneau de gauche montre la distribution d'intensité des impulsions attosecondes (ordres harmoniques 15-30) en champ lointain, générés lorsqu'un champ laser intense $\left(a_{0}=6, w_{f}=8 \lambda_{L}, \tau_{f}=22 \mathrm{fs}, \theta_{L}=112 \mathrm{mrad}\right)$ avec rotation de ses fronts d'onde à la vitesse $v_{r}=v_{r}^{\max }=3.8 \mathrm{mrad} / \mathrm{fs}$, se réfléchit sur le miroir plasma où il induit l'effet ROM. Ces paramètres physiques sont réalistes d'un point de vue expérimental et le rapport entre divergence harmonique et divergence laser, consistant avec les récents résultats expérimentaux obtenus à Saclay sur le laser 100TW UHI100. (b) Le panneau de droite, lui, montre le profil d'intensité du champ en (a), que l'on a intégré angulairement après filtrage spatial par le diaphragme schématisé en rouge sur le panneau de gauche, qui lui, sélectionne approximativement $60 \%$ de l'énergie de l'impulsion principale. La courbe bleue correspond au profil temporel obtenu à partir d'une simulation PIC réalisée dans les mêmes conditions d'interaction, mais sans rotation de front d'onde. Les deux courbes on été normalisées par l'intensité maximum du train sans rotation de front d'onde.

\section{Validation numérique dans les cas de plasmas}

\subsection{Modèle du miroir oscillant (ROM)}

On utilise dans un premier temps le modèle du miroir oscillant développé par Lichters et al. [3], que l'on a généralisé en $2 \mathrm{D}$ pour calculer le champ réfléchi par le miroir plasma en présence d'une rotation du front d'onde de l'impulsion focalisée. Dans ce modèle, la position $Z\left(x_{f}, t\right)$ de la surface du miroir plasma à la position transverse $x_{f}$, effectue une oscillation harmonique à la fréquence du laser, avec une vitesse pic $v_{m} / c=a / \sqrt{1+a^{2}}$, où $a\left(x_{f}, t\right)$ est l'enveloppe spatio-temporelle du potentiel vecteur normalisé du laser incident. Cela conduit $Z\left(x_{f}, t\right)=\left(v_{m} / \omega_{L}\right) \times \cos \varphi\left(x_{f}, t\right)$, avec $\varphi$ donné par l'Eq. (2). Le champ réfléchi $\tilde{E}_{r}\left(x_{f}, t\right)$ est alors proportionnel à $\tilde{E}\left(x_{f}, t_{r e t}\right) e^{i k_{L} Z\left(t_{r e t}\right)}$, où $t_{r e t}\left(x_{f}\right)$ est la solution de $Z\left(x_{f}, t_{r e t}\right)=c\left(t-t_{\text {ret }}\right)$. Ceci rend compte des effets de retards liés à la propagation de la lumière et donc, de l'effet Doppler induit par les oscillations de la surface du miroir plasma. Une fois que l'on a calculé le champ réfléchi $\tilde{E}_{r}\left(x_{f}, z=0, t\right)$ juste après sa réflexion sur la cible, on calcule sa propagation dans le vide en utilisant une décomposition en ondes planes afin de déterminer sa forme $E_{r}(x, z, t)$ à une distance arbitraire $z$ de la cible.

Les prédictions du modèle ROM sont représentées sur la Fig. 4. Comme prévu, en l'absence de rotation $\left(v_{r}=0\right)$ de front d'onde au foyer, des harmoniques de la fréquence laser sont émises dans un seul faisceau collimaté, avec une divergence plus faible que celle de la fréquence fondamentale, et sont associées, dans le domaine temporel, à un train de 5 impulsions attosecondes. Lorsqu'on augmente le tilt du front d'intensité $\xi, v_{r}(\xi)$ augmente au foyer et ce faisceau unique se sépare progressivement en un ensemble de plusieurs faisceaux. À la vitesse de rotation maximale $v_{r}^{\max }$, ces faisceaux sont bien séparés angulairement et chacun d'eux est constitué d'un spectre continu qui est associé, dans le domaine temporel, à une impulsion attoseconde.

Si on continue d'augmenter le tilt du front d'intensité $\xi$ au delà de la valeur optimale $\xi_{0}=\tau_{i} / w_{i}$, on remarque qu'il est est toujours possible de séparer des impulsions à l'aide du phare attoseconde mais à présent l'extension spectrale décroît très rapidement car le chirp spatial au foyer est trop grand 


\section{Web of Conferences}

et diminue considérablement l'intensité laser. Il n'y a donc aucun intérêt à utiliser l'effet du phare dans ce régime.

\subsection{Simulations PIC 2D}

Afin de confirmer les résultats de ce modèle simple, nous avons réalisé des simulations particulaires 2D de la génération d'harmoniques sur un miroir plasma avec une rotation du front d'onde du champ électrique de l'impulsion focalisée, dans le régime relativiste $\left(a_{0}=6\right)$. Ces simulations ont été réalisées à l'aide du code CALDER. Dans le cas présenté Fig. 5, le plasma a une densité maximum de $100 n_{c}$, un gradient de densité initial de $\lambda_{L} / 200$ et une température électronique initiale de $0,1 \mathrm{keV}$. Les ions sont mobiles. L'angle d'incidence du faisceau laser sur la cible vaut $45^{\circ}$ et le champ incident est polarisé $p$. Ce champ est injecté dans la boîte de simulation par l'intermédiaire de conditions aux limites, sous la forme de l'Eq. (2). On propage ensuite le champ réfléchi juste après la cible $\tilde{E}_{r}\left(x_{f}, t\right)$, issu des simulations, à l'aide d'une décomposition en ondes planes. À la vitesse de rotation du front d'onde maximale, on voit clairement l'effet phare attoseconde sur la Fig. 5 (a). Dans ce cas, la vitesse de rotation est suffisamment grande et la divergence du faisceau harmonique suffisamment petite pour pouvoir isoler une seule impulsion attoseconde en plaçant simplement une fente en champ lointain [cf. Fig. 5 (b)]. Ceci montre qu'il est possible de satisfaire le critère de séparation d'une impulsion attoseconde d'un train dans le cas des harmoniques Doppler générées sur miroir plasma.

\section{Références}

[1] H. Vincenti anf F. Quéré, Physical Review Letters 108, 103904 (2012)

[2] S. Akturk, X. Gu, P. Bowlan, and R. Trebino, Journal of Optics 12, 093001 (2010)

[3] R. Lichters, J. Meyer-ter-Vehn, and A. Pukhov, Physics of Plasmas 3, 3425 (1996) 